\title{
ENCALAMIENTO DE UN SUELO SULFATADO ÁCIDO DE CÓRDOBA Y SU EFECTO SOBRE EL DESARROLLO DE TRES GENOTIPOS DE MAÍZ EN INVERNADERO
}

\section{LIMING OF ACID SULPHATE SOILS OF CÓRDOBA AND ITS EFFECT ON DEVELOPMENT OF THREE MAIZE CULTIVARS IN GREENHOUSE}

Enrique Combatt Caballero ${ }^{1}$

Alfredo Jarma Orozco ${ }^{1}$

Liliana Atencio Solano²

\section{RESUMEN}

El ensayo se realizó en invernadero en la Universidad de Córdoba, con el fin de evaluar el efecto del encalamiento sobre el desarrollo de tres genotipos de maíz (Zea mays) y sobre las propiedades químicas de un suelo sulfatado ácido (ssa) del Valle del Sinú. Se utilizó un diseño experimental completamente al azar con arreglo factorial, donde el factor A correspondió a los genotipos comerciales (Corpoica H-108, ICA V-109, SV-1127) y el $\mathrm{B}$ a los niveles de enmienda de cal agrícola $(0,3$, 6 y 9t/ha). Los resultados indicaron que al pasar de 6 a 9 toneladas de cal por hectárea, SV 1127 registró incrementos importantes de biomasa de raíz por planta $(5 \mathrm{~g})$ y altura de planta $(25 \mathrm{~cm})$, en tanto que el área foliar fue significativamente superior en estos dos tratamientos (más de $450 \mathrm{~cm}^{2}$ ), respecto al resto. Estas respuestas no fueron tan evidentes para los otros genotipos, que presentarían un comportamiento más estable en este tipo de suelos. Las respuestas observadas permitieron concluir que la práctica de encalamiento fue eficiente para reducir los niveles de acidez y mejorar la relación $\mathrm{Ca}^{2+} \mathrm{y} \mathrm{Mg}^{2+}$ y bases intercambiables y acidez del suelo. Así mismo, los resultados permitieron concluir que SV-

1 ( I.A. M.Sc. Facultad Ciencias Agrícolas Universidad de Córdoba. ecombatt@sinu.unicordoba.edu.co

2 I.A. Asistente Técnico Particular. lilo10_6@hotmail.com
1127 presentaría mejor adaptación cuando la acidez del suelo es neutralizada, en tanto que Corpoica H 108 sería un genotipo más estable al ser cultivado en este tipo de suelos, ya sean encalados o no.

Palabras clave: Acidez del suelo, aluminio, crecimiento y desarrollo, contenido nutricional, maíz.

\section{SUMMARY}

The research was carried out under greenhouse conditions of the Universidad de Córdoba, with the purpose of evaluating the effect of the lime level on the development of three corn genotypes (Zea mays) and on the chemical properties of an acid sulphate soil (ass) of the river Sinú valley. A complete randomized experimental design was used with factorial arrangement, where factor A corresponded the commercial corn genotypes (Corpoica H-108, ICA V-109, SV-1127) and B to the applied lime levels $(0,3,6$ and $9 \mathrm{t} / \mathrm{ha})$. The results indicated that when passing from 6 to 9 tons of lime per hectare, SV 1127 registered important increases of the root biomass per plant $(5 \mathrm{~g})$ and of the plant height $(25 \mathrm{~cm})$, also the leaf area was significantly superior in these two treatments (more than $450 \mathrm{~cm}^{2}$ ), compared to others. These responses were not so evident for the other genotypes that would present a more stable behavior in this soil type. The results observed allowed to conclude that liming was efficient to reduce the acidity levels and to improve the relationship $\mathrm{Ca}^{2+}$ and $\mathrm{Mg}^{2+}$, 
the interchangeable bases and the soil acidity. Likewise, the results allowed to conclude that SV-1127 would present an enhanced adaptation when the soil acidity is neutralized, mean while Corpoica $\mathrm{H} \mathrm{108,} \mathrm{would} \mathrm{be} \mathrm{a}$ more stable genotype when cultivated in this soil type, limed or not.

Key words: Acid soil, aluminum, growth and development, nutritional content, maize.

\section{INTRODUCCIÓN}

Los suelos sulfatados ácidos (ssa), se presentan en zonas donde se encuentran depósitos de materiales parentales ricos en pirita y se ubican generalmente en áreas planas inundadas y frecuentemente drenadas para la agricultura o la construcción. Cuando se exponen los sulfuros al aire tiene lugar la oxidación que origina finalmente una alta acidez que excede la capacidad de neutralización de los mismos. En algunos casos disuelve también los metales pesados que pueden ser tóxicos para la mayoría de las plantas (Dent, 1986; Graham E Larsen, 2000; Sammut, 2002).

Se estima que más de 200 millones hectáreas en el mundo se encuentran cubiertas por turbas, las cuales pueden originar ssa; de éstas, alrededor de 32 millones están en el trópico y un poco más del $80 \%$ están formadas en tierras costeras bajas (Fitzpatrick, 1996). Estudios realizados en estas zonas por Dent $\mathcal{E}$ Dawson (2000), mediante sistemas informáticos, estiman un total de 24 millones de hectáreas de ssa en el mundo.

En Colombia no hay una cuantificación detallada del área cubierta con ssa, pero ocurren con relativa frecuencia en los departamentos de Sucre, Bolívar, Boyacá, Valle del Cauca y Córdoba. En este último departamento existen entre 10.000 y 20.000 ha, en las cuales el drenaje y el secamiento de las zonas de bacines de la llanura aluvial dirige los procesos hacia la acidificación y la formación de ssa interiores (Combatt, 2004).

Los ssa reducen considerablemente la productividad agrícola, lo que se ve reflejado en el estrés fisiológico de los cultivos, debido a que la acidez soluble, entre otros efectos, desciende el $\mathrm{pH}$ a valores menores de 4 , de tal forma que las altas concentraciones de iones hidrógeno en la solución del suelo, promueven el ataque a las estructuras cristalinas de las arcillas, lo que permite que se liberen iones de aluminio a la solución (Sammut, 2002).

En plantas susceptibles, la acumulación de $\mathrm{Al}^{+3}$ es más intensa en las raíces. Las lesiones radiculares provocadas por la toxicidad de ese elemento causan desorganización de las estructuras y de las funciones de las membranas, parálisis de la síntesis de ADN y de la mitosis, reducción del elongamiento celular al ligarse con pectinas, disturbios en la asimilación y en el metabolismo mineral de los ápices de las raíces y disminución de la respiración radical (Pereira et al. 2007; Mossor, 2001).

Diversos autores han indicado que la acidez se puede corregir mediante el encalado, neutralizando los iones hidrógeno de la solución del suelo con los iones hidroxilo que se producen al aplicar los materiales encalantes (Ward et al. 2002; Guimaraes et al. 2000). En el caso del carbonato de calcio, se disuelve a medida que los iones hidroxilo se remueven de la solución del suelo, produciendo iones calcio y bicarbonato. Otros investigadores, como Delhaize \& Ryan (1995) y Kochian (1995), indican que las estrategias para aumentar la producción en suelos ácidos incluyen, además del uso de plantas tolerantes a la acidez, la aplicación de cal, ya que la aplicación de calcio o de carbonato de calcio reduce el estrés causado por el aluminio, al ser neutralizado mediante la precipitación o la insolubilización con los hidroxilos producidos en la disociación del carbonato de calcio y el agua. No obstante a lo anterior, Sierra et al. (2003) mencionan que la práctica del encalamiento es a menudo costosa y poco eficiente en muchas partes de los trópicos y Mathew et al. (2001) recomiendan realizar lavados antes de encalar, para posteriormente, fertilizar.

Por otra parte, el desarrollo de muchos genotipos de maíz tolerantes al aluminio ha contribuido grandemente al incremento de la productividad de su biomasa en suelos ácidos. Los mecanismos de esta adaptación, aunque parcialmente desconocidos, pueden ser explicados de manera general por la exclusión del aluminio por la raíz vía citratos (Piñeros et al. 2005) y/o por la inactivación interna de éste. En este sentido, diversos autores han concluido que aunque los mecanismos de exclusión del aluminio en algunas especies pueden no estar completamente identificados es conocido que muchas 
de ellas tienen mecanismos eficientes para la exudación de este ión a través de ácidos orgánicos por las raíces (Watanabe E Osaki, 2002; Taylor et al. 2000).

Considerando lo anterior y en razón a las áreas crecientes de ssa en el bajo Sinú (Córdoba), se planteó la investigación bajo condiciones de invernadero, con el objetivo de evaluar el efecto del encalamiento sobre las propiedades químicas de este tipo de suelos y sobre el crecimiento y el desarrollo de tres genotipos comerciales de maíz (Zea mays L.).

\section{MATERIALES Y MÉTODOS}

El ensayo se realizó en un invernadero de la Universidad de Córdoba, (Montería - Colombia), ubicada a $8^{\circ} 75^{\prime}$ de latitud norte y $75^{\circ} 55^{\prime}$ de longitud oeste, a una altura de $15 \mathrm{msnm}$., HR del $85 \%$ y temperatura promedio $28^{\circ} \mathrm{C}$.

Se utilizó un suelo con régimen de temperatura Isohipertérmico clasificado como Typic Sulfaquepts, con una pendiente del 1- $2 \%$, ubicado en la cubeta de decantación, drenaje externo muy lento, interno encharcado, con presencia de sedimentos aluviales, cuya limitante es el horizonte sulfúrico. Luego de tomar las muestras para el análisis químico inicial, el suelo se colocó en bolsas de polietileno en cantidad de $12 \mathrm{~kg}$ por bolsa, conservando la secuencia encontrada en campo de los primeros $40 \mathrm{~cm}$ del perfil. Con base en este volumen, se estimaron las dosis de 3, 6 y 9 toneladas de cal agrícola por hectárea en g por bolsa (432, 864 y 1296, respectivamente). Los análisis químicos del suelo, se determinaron de acuerdo a la metodología del Instituto Geográfico Agustín Codazzi (1990).

Para el ensayo, se utilizó un diseño completamente al azar con arreglo factorial de 3 x 4 y cuatro repeticiones, donde el factor A estuvo conformado por los genotipos (Corpoica H-108, ICA V-109 y SV-1127) y el factor B por las dosis de cal agrícola $\left(\mathrm{CaCO}_{3}\right)$ del $90 \%$ de pureza $(0,3,6$ y 9t/ha). Después de 30 días del encalado (dde), se sembraron los genotipos de maíz. La fertilización con nitrógeno, fósforo y potasio, se realizó de acuerdo a los resultados del análisis químico para cada uno de estos elementos, en cantidades equivalentes por hectárea, como se relacionan a continuación: fósforo en presiembra incorporado a razón de $46 \mathrm{~kg}$, como fosfato diamónico; nitrógeno, como urea en dosis de $92 \mathrm{~kg}$ y potasio, como $\mathrm{KCl}$ en razón de 60kg. Cada unidad experimental estuvo constituida por 10 plantas (una en cada bolsa), aplicándola un volumen de riego de 5L por bolsa cada tres días, con base en las tasas de evapotranspiración observadas en la región. Con el propósito de observar la dinámica de los elementos químicos, a los 35 y 75 días después de la siembra (65 y 105dde), nuevamente se tomaron muestras de suelo. Las variables fisiológicas masa seca de la raíz, altura de planta $(\mathrm{cm})$ y área foliar $\left(\mathrm{cm}^{2}\right)$, se tomaron mediante muestreo destructivo sobre cinco plantas por tratamiento a los 75dde.

Para el análisis estadístico de los datos, se aplicaron estudios de varianza. Cuando se presentaron interacciones, se llevaron a cabo comparaciones horizontales para cada variedad dentro de las dosis y cuando éstas no se detectaron, se realizaron pruebas de comparación de medias, según la Prueba de Comparación Múltiple de Tukey $(\mathrm{P}<0,05)$.

\section{RESULTADOS Y DISCUSIÓN}

\section{Análisis químico del suelo.}

Inicial. Las condiciones iniciales del suelo indican que el $\mathrm{pH}$ es fuertemente ácido, la materia orgánica se considera como alta para la región, el azufre es excesivo y el fósforo medio. El calcio, el magnesio y el potasio presentan niveles altos (relación 27,5), en tanto que el sodio, al nivel observado, no causaría problemas. La concentración de aluminio, se puede considerar como excesiva (Cuadro 1).

65 y 105 días después de encalado. Los resultados del análisis indican que, en cualquiera de las dos épocas de evaluación, el pH del suelo muestra una tendencia de aumento en la medida que se incrementa la dosis de cal, superando los valores iniciales entre un 27 y $33 \%$ a los $65 d d e$ y entre un 27 y $43 \%$ a los $105 d d e$. Esto estaría de acuerdo con lo reportado por Zhang et al. (2004), quienes afirman que el $\mathrm{pH}$ incrementa con el tiempo y con las dosis de cal aplicadas.

A los 65dde, la materia orgánica continúa siendo alta sin que se presente un cambio considerable en su porcentaje, lo que probablemente sea debido a la poca actividad de los microorganismos, que en esta época aún continuarían siendo escasos. Sin embargo, a los 105dde estos valores disminuyen, eventualmente, como 
Cuadro 1. Caracterización química de suelos sulfatados ácidos a los 65 y 105 días después de tres niveles de encalamiento.

\begin{tabular}{|c|c|c|c|c|c|c|c|c|c|}
\hline Tratamiento & pH & MO & S & P & Ca & Mg & K & Na & Al \\
\hline & 1,1 & $\%$ & $\mathrm{mg} / \mathrm{kg}$ & \multicolumn{7}{|c|}{$\mathrm{cmol}_{\mathrm{c}} \mathrm{kg}^{-1}$} \\
\hline T0 & 3,56 & 7,43 & 1250 & 47,09 & 6,8 & 10,8 & 0,64 & 0,55 & 2,6 \\
\hline \multicolumn{10}{|c|}{65 días después de encalado } \\
\hline T1 & 4,51 & 7,24 & 737,6 & 24,5 & 19 & 7,5 & 0,6 & 0,7 & 0,8 \\
\hline T2 & 4,54 & 8,31 & 622,7 & 24,5 & 18,5 & 5 & 0,8 & 0,6 & 0,7 \\
\hline T3 & 4,74 & 6,21 & 955,5 & 22 & 18,5 & 8 & 0,7 & 0,8 & 0,4 \\
\hline \multicolumn{10}{|c|}{105 días después de encalado } \\
\hline T1 & 4,54 & 6,21 & 890,9 & 23,6 & 17 & 9 & 0,9 & 0,8 & 0,7 \\
\hline T2 & 4,54 & 6,21 & 487,5 & 33,6 & 21,5 & 4 & 0,7 & 0,7 & 0,6 \\
\hline T3 & 5,12 & 5,17 & 1056,4 & 26,2 & 19 & 8,5 & 1 & 0,9 & 0,4 \\
\hline
\end{tabular}

T0=Testigo absoluto sin encalar; T1, T2 y T3=Tratamientos con 3, 6 y 9 toneladas de cal por hectárea.

una consecuencia de la mineralización, ocasionada por las primeras actividades microbianas en condiciones químicamente mejores, lo que estaría acorde con lo reportado por diversos autores, quienes dicen que el encalado favorece la mineralización de la materia orgánica, ya que proporciona a los microorganismos no tolerantes a la acidez, un ambiente favorable para su desarrollo (Sadzawka \& Campillo, 1993; Neale et al. 1997), resultando un incremento de la biomasa microbial y la respiración del suelo, al igual que la mineralización del nitrógeno por la liberación de materia orgánica poco estable en la forma de compuestos orgánicos disueltos (Tate, 2000; Fuentes et al. 2006).

El comportamiento del azufre sugiere que a pesar de observarse una disminución en sus niveles, en cualquiera de los dos momentos de observación, se presenta aún en concentraciones excesivas; de manera independiente al nivel de cal utilizado, los contenidos de fósforo se reducen en un 50 y $41 \%$ a los 65 y 105dde, respectivamente, considerándose estos valores como medios.

Calcio, magnesio y potasio tienen altas concentraciones intercambiables sin presentar una tendencia definida en función del tiempo y nivel de encalamiento; la relación de estos elementos varió de 27,5 en el suelo sin encalar a alrededor de 34 en promedio para el suelo encalado; sodio, aunque registra una leve tendencia a aumentar con estas variables, sus valores no causarían problemas de toxicidad (Grupta \& Abrol, 1990).

La acidez intercambiable $\left(\mathrm{Al}^{3+}+\mathrm{H}^{+}\right)$, se reduce significativamente tanto a los 65 como a los 105dde, con el nivel de cal aplicado, aunque lo hace de manera más fuerte al aplicar 9t de cal por hectárea. Lo anterior, se podría explicar por el efecto de la neutralización de este elemento al precipitarse como hidróxido de aluminio, concordando con estudios en los que se indica que el encalamiento presenta efectos positivos al corregir la acidez en el suelo y reducir la toxicidad por aluminio (Guimaraes et al. 2000).

\section{Aspectos fisiológicos de la planta.}

El cuadro 2 registra los cuadrados medios de las variables masa seca de raíz, altura de planta y área foliar con su respectiva significancia estadística, indicando que existe un fuerte efecto de la interacción genotipo por dosis en todas las variables fisiológicas evaluadas.

Masa seca de raíz. Al analizar los resultados de la biomasa de raíz por planta se pudo determinar que el 
Cuadro 2. Cuadrados medios del análisis de varianza para variables fisiológicas de tres genotipos de maíz y cuatro dosis de cal en ssa a los 75 dds.

\begin{tabular}{|l|c|c|c|c|}
\hline FUENTE DE VARIACIÓN & GL & $\begin{array}{c}\text { Masa Seca Raíz } \\
\mathbf{g}\end{array}$ & $\begin{array}{c}\text { Altura de Planta } \\
\mathbf{c m}\end{array}$ & $\begin{array}{c}\text { Área Foliar } \\
\mathbf{c m}^{2}\end{array}$ \\
\hline Genotipos & 2 & $913,640^{* *}$ & $1911,000{ }^{* *}$ & $44086933^{* *}$ \\
\hline Dosis & 3 & $533,356^{* *}$ & $265,5833 \mathrm{~ns}$ & $759594^{* *}$ \\
\hline Genotipo x Dosis & 6 & $155,020^{* *}$ & $747,6667^{*}$ & $458478^{*}$ \\
\hline Residuo & 24 & 8,9522 & 283,0833 & 138252 \\
\hline C.V. (\%) & & 12,4 & 13,45 & 14,71 \\
\hline Desvío Estándar & & 11,42 & 21,31 & 700,30 \\
\hline
\end{tabular}

${ }^{*} \mathrm{y}{ }^{* *}=$ Significativos al 0.05 y $0.01 \%$ respectivamente de acuerdo al test de Tukey. ns $=$ No significativo $(0.05 \%)$

comportamiento de las variedades ante diferentes niveles de saturación de aluminio es diferencial.

La Figura 1 muestra el análisis de la interacción y evidencia la tendencia a aumentar de la masa seca de raíz conforme aumentan las dosis de cal para los genotipos SV-1127 e ICA V-109, contrario al comportamiento de Corpoica $\mathrm{H}-108$ (C H 108), que tiende a mantener una biomasa de raíz relativamente estable a pesar de aumentarse las dosis de cal. El comportamiento de C H 108 explica la interacción y sugiere la capacidad de este genotipo de soportar altos niveles de acidez sin afectar el desarrollo de sus raíces, coincidiendo con lo reportado por sus creadores al indicar que la variedad puede tolerar hasta un $70 \%$ de saturación de aluminio (Corpoica, 2007).

Es posible que la formación de complejos orgánicos, como exudados radiculares para impedir la entrada del aluminio, favorezca la toma de nutrientes esenciales y no afecte significativamente la masa seca de la raíz en C H 108 a los niveles de acidez evaluados, tal lo han enunciado varios autores en otras especies (Taylor et al. 2000; Marschner, 2002; Casierra, 2001; Caires et al., 2002). Por otra parte Horst (2005) y Jorge \& Arrudat (1997) explican que existen variedades de maíz tolerantes a la acidez por su capacidad de excretar compuestos orgánicos, como el citrato, que permiten detoxificar el aluminio, permitiendo mejorar la disponibilidad del fosfato y excluir la toxicidad del ión de las células del ápice de la raíz de plantas tolerantes, que se pueden ver afectadas por el ambiente.
Por otra parte, el aumento de biomasa de raíz en SV-1127 e ICA V-109 al aumentar la neutralización de la acidez por el encalamiento, se puede deber a que la susceptibilidad de estos materiales a altos niveles de acidez (expresada en términos de biomasa de raíz), se reduce significativa y progresivamente, como consecuencia, probablemente, de una mayor disponibilidad de elementos, como el fósforo que al ser constituyente fundamental de moléculas energéticas, como el ATP y ADP, entre otras, favorece el desarrollo y la formación de las raíces, de tal manera que el sistema radical sea más vigoroso y extenso (Rincón et al., 2003). Así mismo, el fósforo junto con el nitrógeno y el calcio en las células permiten que los procesos de división y de elongación se aceleren, favoreciendo la ramificación y la proliferación de las raíces. Es probable que el menor desarrollo radicular de SV 1127, observado en el tratamiento donde no se neutralizó la acidez, se pueda deber al bloqueo de la expansión celular inducida por el aluminio, más que a la división celular, tal como lo propusieran Martínez-Esteves E Ku-Gonzalez (2003) y Mossor (2001) para café.

Altura. Consecuentemente con los resultados observados para la biomasa de raíz, los genotipos mostraron una altura de planta de forma diferencial ante las dosis de cal, siendo explicada esta interacción por el comportamiento de SV 1127 que tiende a aumentar su altura de manera significativa $(P<0,05)$ cuando se aplican $9 t$ de cal/ha, sugiriendo que este material responde de manera positiva al reducirse los niveles de acidez del suelo (Figura 2). Esta situación no fue tan evidente para C H 108 e ICA V 109 , los cuales no presentan una tendencia definida en 


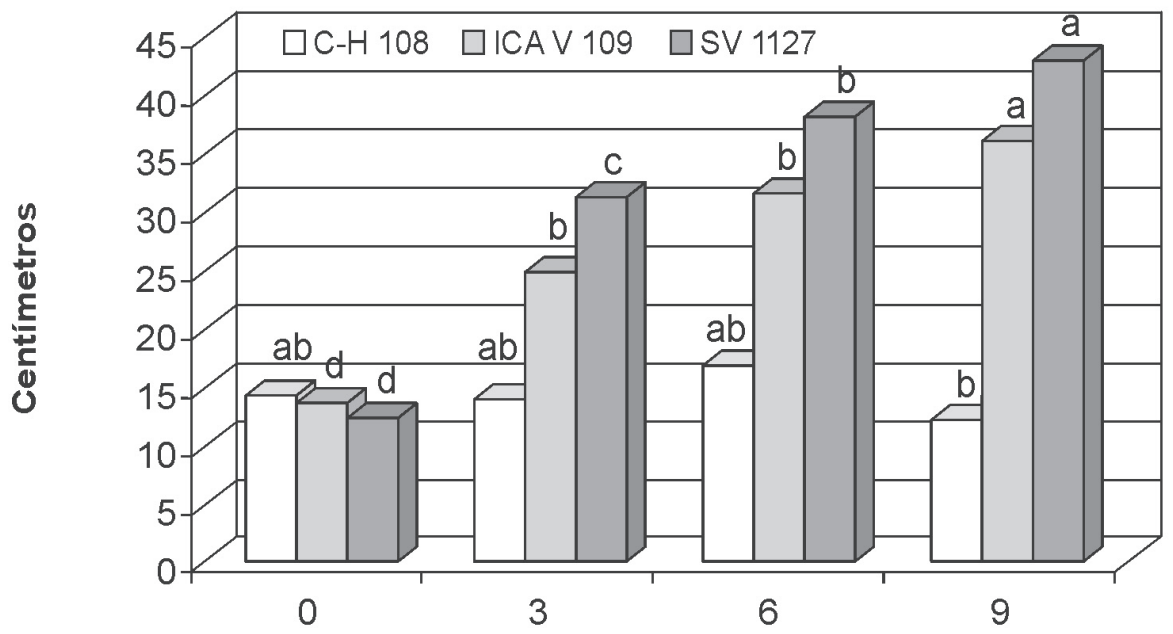

Toneladas de cal por hectárea

Promedios dentro de un genotipo (columnas del mismo color) que presentan la misma letra, no difieren estadísticamente a un nivel del $5 \%$ de probabilidad de acuerdo a test de Fisher-LSD.

Figura 1. Interacción dosis $\mathrm{x}$ genotipo para biomasa de raíz de tres genotipos de maíz bajo cuatro niveles de encalamiento de ssa en Córdoba.

la medida que aumentan las dosis de cal y sugieren una mejor adaptación genética a las condiciones de los ssa evaluados, lo que se puede explicar por el comportamiento diferencial entre especies y genotipos dentro de una especie, respecto a sus requerimientos nutricionales y la oferta de estos en la rizosfera (Meléndez et al. 2006).

Los valores registrados de altura de planta en SV 1127 pueden ser una consecuencia del mayor crecimiento radicular en el ambiente donde los niveles de acidez no fueron limitantes por el efecto de la cal, al permitir una mayor área de exploración y mejorar la eficiencia en la captación de otros elementos, como se discutió en el ítem anterior y como lo afirman Meléndez et al. (2006), al indicar que el crecimiento de la planta está influenciado, entre otros factores, por la disponibilidad de nutrientes en el ambiente radicular. Las positivas correlaciones entre desarrollo radicular y crecimiento del dosel, así como la importancia de cuantificar el crecimiento de raíces para un mejor entendimiento del desarrollo de toda la planta, han sido confirmadas por varios investigadores en diversas especies, como papa, maíz y vid (ÁlvarezSánchez et al. 2007; Pire E Díez, 2006).

Es probable que la mayor altura de planta registrada por SV 1127, como consecuencia de una mayor disponibilidad de nutrientes en la raíz en los tratamientos donde se neutralizó la acidez, haya obedecido a mayores tasas de producción de asimilados, las cuales podrían ser una consecuencia de una mejor eficiencia fotosintética, que sería debida a la mayor disponibilidad de elementos como el $\mathrm{Fe}^{2+}$ y $\mathrm{Mg}^{2+}$, elementos fundamentales en la síntesis de moléculas fotosensibles, como fitocromos y clorofilas, respectivamente, a partir de su adición a la protoporfirina IX en la ruta metabólica que se origina a partir del ácido glutámico. Esta teoría sería coincidente con lo reportado por Villar E Ortega (2003), quienes reportaron que los niveles de clorofila se ven negativamente afectados por condiciones adversas, como la deficiencia en la disponibilidad de nutrientes.

Área foliar. La descomposición de la interacción indicó que la misma está nuevamente explicada por el comportamiento diferencial de SV 1127, al aumentar significativamente su área foliar, cuando no tiene niveles limitantes de acidez en el suelo, tal como lo registra la Figura 3. El mayor desarrollo de la raíz y del dosel de este genotipo bajo estas condiciones, como consecuencia de una mayor disponibilidad de nutrientes y de pigmentos fotosensibles (Costa et al. 2001; Novoa E Villagrán, 2002), le permitiría desarrollar una mayor área para la captación de luz (Otero E Polanía, 2002), ya sea por la expansión de las hojas 


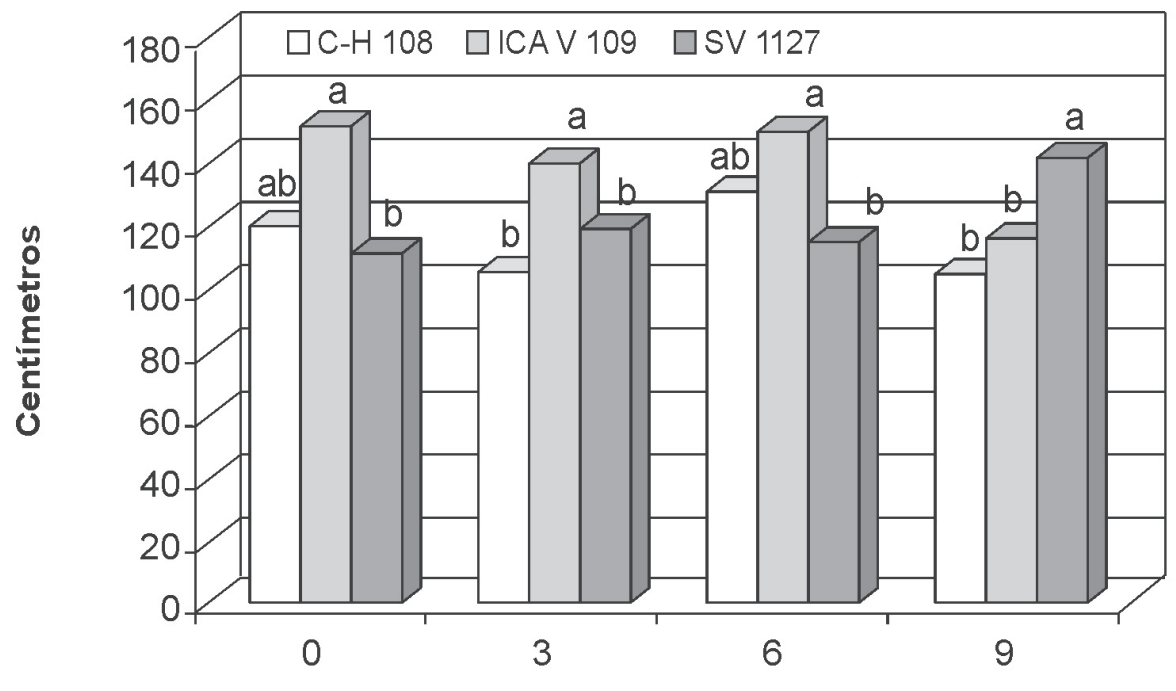

Toneladas de cal por hectárea

Promedios dentro de un genotipo (columnas del mismo color) que presentan la misma letra, no difieren estadísticamente a un nivel del $5 \%$ de probabilidad de acuerdo a test de Fisher-LSD.

Figura 2. Interacción dosis x genotipo para altura de planta de tres genotipos de maíz bajo cuatro niveles de encalamiento de ssa en Córdoba.

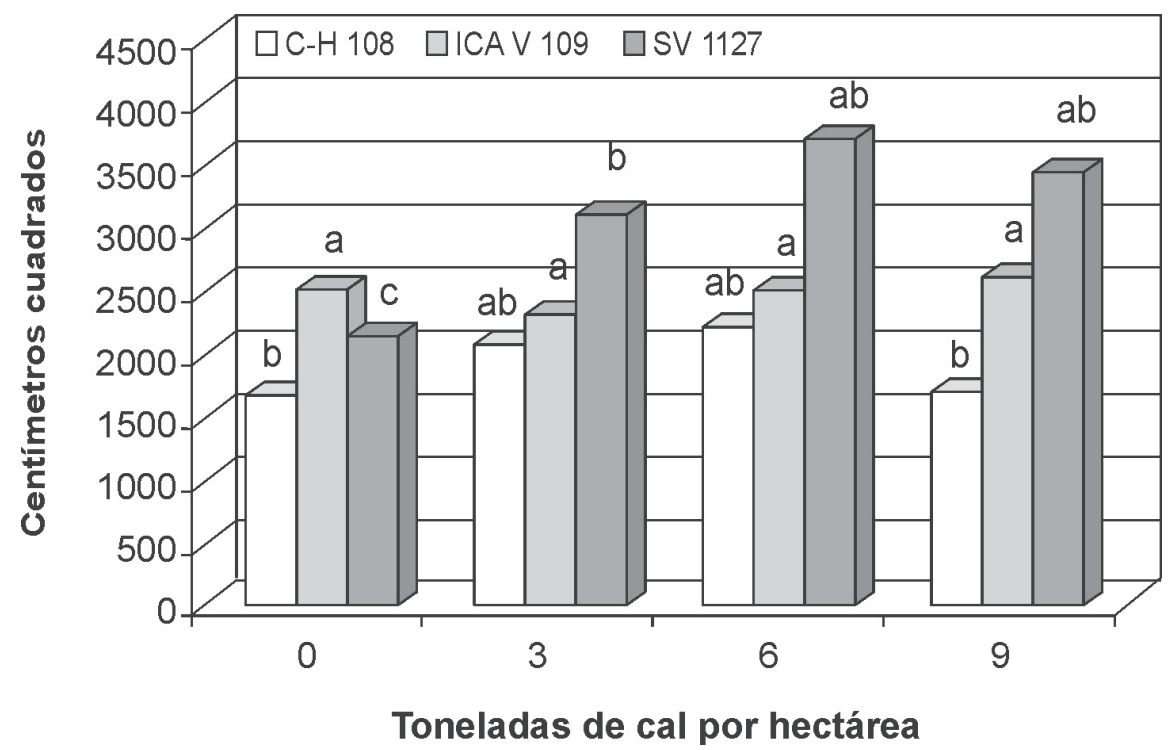

Promedios dentro de un genotipo (columnas del mismo color) que presentan la misma letra, no difieren estadísticamente a un nivel del $5 \%$ de probabilidad de acuerdo a test de Fisher-LSD.

Figura 3. Interacción dosis x genotipo para área foliar por planta de tres genotipos de maíz bajo cuatro niveles de encalamiento de ssa en Córdoba.

(Fredeen et al. 1989) y/o por el mayor número de estas (Lynch et al. 1991); esto se podría traducir en mayores tasas de conversión de fotoasimilados y, finalmente, en una mayor acumulación de biomasa en toda la planta, tal como se discutió y de manera consecuente con la altura de la planta. Sin embargo, aunque se registraron menores valores de área foliar en C H 108, consecuencia de su menor desarrollo del dosel y biomasa de raíz, los 
resultados sugieren que este material tiende a mantener sus características de crecimiento estables a pesar de variar las condiciones de acidez del suelo, desarrollando un dosel acorde con su biomasa radicular.

Por otra parte, a pesar que ICA V 109 muestra mayor crecimiento radicular cuando se aplican altas dosis de cal por hectárea esta condición no se refleja en mayores tasas de crecimiento del dosel como se esperaría, lo que sugiere que este genotipo presenta una menor eficiencia en el intercambio balanceado de la raíz y el dosel (fuente - vertedero), cuando las características químicas del suelo, principalmente la acidez, son variables. Gallardo et al., habían indicado en 2006, que el efecto fitotóxico del aluminio, que se manifiesta principalmente en el sistema radical, también afectaba la parte aérea, afirmación que sin duda se podría asumir como una consecuencia lógica; sin embargo, con base en el desarrollo de ICA V 109 , en el presente ensayo, adicionalmente a lo anterior, se deben considerar las particularidades del genotipo y del nivel de toxicidad.

\section{CONCLUSIONES}

Aunque la cal aplicada no neutralizó la acidez intercambiable, se observó una ligera tendencia de la reacción del suelo a aumentar en la medida que se incrementaron las dosis de cal.

El genotipo que mostró la mejor adaptación a las condiciones del suelo sulfatado ácido evaluado a nivel de invernadero fue $\mathrm{C} \mathrm{H} \mathrm{108,} \mathrm{dada} \mathrm{su} \mathrm{estabilidad} \mathrm{ante} \mathrm{las}$ variaciones de acidez y de disponibilidad de nutrientes. Por su parte, SV 1127 muestra mejor comportamiento fisiológico cuando la acidez no es limitante.

ICA V 109 registra buen desarrollo de raíz al neutralizar la acidez con $9 \mathrm{t}$ de $\mathrm{cal} / \mathrm{ha}$, pero esta característica no es reflejada en el desarrollo del dosel (altura de planta y área foliar).

\section{AGRADECIMIENTOS}

Los autores expresan sus agradecimientos al Centro de Investigaciones de la Universidad de Córdoba CIUC, por el apoyo financiero para el desarrollo del trabajo.

\section{BIBLIOGRAFÍA}

ÁLVAREZ-SÁNCHEZ, E.; ETCHEVERS, B.; ORTIZ, J. 2007. Localización del fertilizante fosfatado y la nutrición de plántulas. REDEPAPA - Red electrónica de la papa. Disponible desde Internet en: http://www.redepapa.org/alvarezsanchez.pdf. (con acceso 12/08/07).

CAIRES, E..; FELDHAUS, I.; BARTH, G.; GARBUIO, F. 2002. Lime and gypsum application on the wheat crop. Scientia Agricola, 59: 357-364.

CASIERRA, F. 2001. Fundamentos fisiológicos, bioquímicos y anatómicos del estrés por aluminio en los vegetales. Revista COMALFI: 28(2):8-19.

COMBATT, E. 2004. Efecto del encalamiento y el lavado sobre algunas propiedades químicas de un suelo sulfatado ácido magnésico del Valle del Rio Sinú. Tesis M.Sc., Universidad Nacional de Colombia, Bogotá. 180p.

CORPOICA - Corporación Colombiana de Investigaciones Agropecuarias. 2007. Híbrido de maíz amarillo Corpoica H 108®. Disponible desde Internet en: http://www.corpoica.org.co/Libreria/verpublicacion.asp?id_publicacion $=1377$. (con acceso 03/08/07).

COSTA, C.; DWYER, P.; DUTILLEU, D.; STEWART, B.; SMITH, D. 2001. Inter-relationships of applied nitrogen, spad, and yield of leafy and non-leafy maize genotypes. J. Plant Nutr. 24(8):1173-1194.

DELHAIZE, E.; RYAN, P. 1995. Aluminum Toxicity and Tolerance in Plants. Plant Phys. 107: 315-321.

DENT, D. 1986. Acid Sulfate Soils: a baseline for research and development, ILRI Publ., No. 39, International Institute for Land Reclamation and Improvement, Wageningen, The Netherlands. 32p.

DENT, D.; DAWSON, B. 2000. The acid test. Disponible en: http://staffi.lboro.ac.uk/ cobrd/. (con acceso 07/08/05).

FITZPATRICK, R. 1996. Acid sulfate soil assessment. Disponible desde Internet en: http://ews.spirit.net. 
Au:8080/audit/full/30-themes-andproyets/50-coping proyec ts/04methods-paper/09-fitzpatrik/acidsulfateconditions.html. (con acceso 13/04/05.

FREDEEN, A.; RAO, I.; TERRY, N. 1989. Influence of phosphorus nutrition on ground and carbon partitioning in Glycine max. Plant Physiol. 89:225-230.

FUENTES, J.; BEZDICEK, D.; FLURY, M.; ALBRECHT, S. y SMITH J. 2006. Microbial activity affected by lime in a long-term no-till soil. Soil $\&$ Tillage Research. 88: 123-131.

GALLARDO, F.; PINO, M; ALVEAR, M. y BORIE, F. 2006. Efecto del aluminio en la producción de materia seca y en la actividad nitrato reductasa de dos variedades de trigo, creciendo en soluciones nutritivas. Universidad de La Frontera. Temuco. Chile. Disponible desde Internet en: http://mingaonline.uach.cl/pdf/rcsuelo /v5n1/art05.pdf. (con acceso 22/01/0/).

GRAHAM, T.; LARSEN, R. 2000. Coastal geomorphology: progressing the understanding of acid sulfate soil distribution. In: Acid sulfate soils: environmental issues, assessment and management. Technical papers. Ahern CR Hey KM, Wating KM and Eidershaw VJ (eds), Brisbane, p.20-22.

GRUPTA, R. y ABROL, I. 1990. Salt affected soils: Their reclamation and management for crop production. Advances of soil science. 11:223-228.

GUIMARAES, E.; SANZ, I.; RAO, I.; AMEZQUITA, M.; AMEZQUITA, E. 2000. Sistemas agropastoriles en sabanas tropicales de América Latina. Cali. Publicación CIAT, No 313, p.16-17.

HORST, W. 2005. Agronomía: Ed. Especial Julio 2005. Disponible desde Internet en: http://europa. eu.int\%00/comm/research/rtdinfo/special_inco/05/ article_2842_es.html. (acceso 18/04/06).

I.G.A.C. 1990. Manual de métodos analíticos de laboratorio de suelos. Subdirección agrológica. V. Edición. Bogotá: IGAC.

JORGE, R.; ARRUDAT, P. 1997 Aluminium-induced organic acids exudation by roots of an aluminium-tolerant tropical maize. Phytochem. 45(4):675-681.
KOCHIAN, L. 1995. Cellular mechanisms of aluminum toxicity and resistence in plant. Ann.. Rev. Plant Physiol. Plant Mol. Biol. 46:237-260.

LYNCH, J.; LAUCHLI, A.; EPSTEIN, E. 1991. Vegetative ground of the common bean in response to phosphorus nutrition. Crop Sci. 31: 380-387.

MARSCHNER, H. 2002. Mineral nutrition of higher plants. London, UK. 616p.

MARTÍNEZ-ESTÉVES, M.; KU-GONZÁLEZ, A. 2003. Changes in some characteristics between the wild and Al-tolerant coffee (Coffea arabica L.) cell line. J. Inorganic Biochem. 97:69-78.

MATHEW, E.; PANDA, R.; NAIR, M. 2001. Influence of subsurface drainage on crop production and soil quality in a low-lying acid sulphate soil. Agr. Water Management 47:191-209.

MELÉNDEZ, L.; HERNÁNDEZ, A.; FERNÁNDEZ, S. 2006. Efecto de la fertilización foliar y edáfica sobre el crecimiento de plantas de maíz sometidas a exceso de humedad en el suelo. Bioagro 18(2):107-114.

MOSSOR, T. 2001. Effect of aluminium on plant growth and metabolism. Acta Bioquímica Polónica. 48(3) 673-686.

NOVOA, R.; VILLAGRÁN, N. 2002. Evaluación de un instrumento medidor de clorofila en determinación de niveles de nitrógeno foliar en maíz. Agr. Técnica 62(1):166-171.

NEALE, S., SHAH, Z. y ADAMS, W. 1997. Changes in microbial biomass and nitrogen turnover in acidic organic soils following liming. Soil Biol. Biochem. 29 : 1463-1474.

OTERO, P.; POLANIA, F. 2002. El cultivo de maíz en Córdoba. Ministerio de Agricultura y Desarrollo Rural PROAGRO. Fondo de Fomento Cerealista y Leguminosas de Grano Fenalce, Bogota, p. 14-16.

PEREIRA, P.; SALES, D. Y CAMBRAIA, J. 2007. Alterações morfológicas e acúmulo de compostos 
fenólicos em plantas de sorgo sob estresse de alumínio. Bragantia (Campinas). 66(1):17-25.

PIÑEROS, M.; SHAFF, J.; MANSLANK, H.; CARVALHO, V.; KOCHIAN, L. 2005. Aluminium resistance in maize cannot be solely explained by root organic acid exudation. A comparative physiological study. Plant Physiology 137: 231-241.

PIRE, R.; DÍEZ, J. 2006. El crecimiento de las raíces de vid en la condición de trópico $\left(10^{\circ} 01^{\prime} \mathrm{N}\right)$ de Tarabana, Estado Lara, Venezuela. Rev. Fac. Agron. 23:453-463.

RINCÓN, J.; GALLARDO, Y.; LEAL, M. y ROJAS, Y. 2003. Efecto de la relación calcio:fósforo en el suelo sobre el crecimiento y nodulación de plantas jóvenes de Acacia mangium (Willd). Bioagro 15(2):97-105.

SADZAWKA, A.; CAMPILLO, R. 1993. Problemática de la acidez de los suelos de la IX región. Manejo del encalado y sus implicancias. IPA Carillanca, 12(3):8-12.

SAMMUT, J. 2002. An introduction to acid sulfate soils NSW Agriculture. NSW. School of Geography. Disponible desde Internet en: http://www.agric.nsw. gov.au/reader/ 10742. (con acceso 24/08/04).

SIERRA, J.; NOEL, C.; DUFOUR, L.; OZIER-LAFONTAINE, H.; WELCKER, C.;DESFONTAINES, L. 2003. Mineral nutrition and growth of tropical maize as affected by soil acidity. Plant Soil 252:215-226.
TATE, R. 2000. Soil Microbiology. En: New York. John Wiley, ESons. 2a ed.508 p.

TAYLOR, G.; MCDONALD-STEPHENS, J.; HUNTER, D.; BERTSCH, P.; ELMORE, D.; RENGEL, Z.; REID, R. 2000. Direct measurement of aluminum uptake and distribution in single cells of Chara corallina. Plant Physiol. 123:987-996.

VILLAR, D.; ORTEGA, R. 2003. Medidor de clorofila. Bases Teóricas y su aplicación para la fertilización nitrogenada en cultivos. Rev. Agr. Forest. UC 18(5):4-8.

WARD, N.; SULLIVAN, L.; BUSH, R. 2002. Sulfide oxidation and acidification of acid sulfate soil materials treated with $\mathrm{CaCO} 3$ and seawaterneutralized refinery residue. Aust. J. Soil Res. 40:1057-67.

WATANABE, T.; OSAKI, M. 2002. Mechanisms of adaptation to high aluminum condition in native plant species growing in acid soils: a review. Com. Soil Sci. Plant Analysis. 33:(7 y 8), 1247-1260.

ZHANG, H.; SCHRODER, J.; KRENZER, E.; KACHURINA, O.; PAYTON, M. 2004. Yield and Quality of Winter Wheat Forage as Affected by Lime. Disponible desde Internet en: http://www.plantmanagementnetwork. org/pub/fg/research/2004/lime. (con acceso 12/08/07).

Recibido: Septiembre 26 de 2007

Aceptado: Noviembre 9 de 2007 